\title{
Direct aspiration stroke thrombectomy: a comprehensive review
}

\author{
William Boisseau, ${ }^{1}$ Simon Escalard, ${ }^{1}$ Robert Fahed (1) , ${ }^{1,2}$ Bertrand Lapergue, ${ }^{3}$ \\ Stanislas Smajda (1) ,' Benjamin Maier, ${ }^{1,4}$ Jean Philippe Desilles, ${ }^{1,4}$ \\ François Delvoye (D) ,' Gabriele Ciccio, ${ }^{1}$ Hocine Redjem, ' Solène Hebert, ${ }^{1}$ \\ Malek Ben Maacha, ${ }_{1}^{1}$ Gregory Walker, ${ }^{2,5}$ Benjamin Gory (D) , ${ }^{6}$ Sébastien Richard, ${ }^{7}$ \\ Mikael Mazighi, ${ }^{1,4}$ Michel Piotin (D) , ${ }^{1,4}$ Raphaël Blanc (D) 1,4
}

- Additional material is published online only. To view please visit the journal online (http://dx.doi.org/10.1136/ neurintsurg-2019-015508).

For numbered affiliations see end of article.

\section{Correspondence to}

Dr Raphaël Blanc, Departement of interventional neuroradiology, Fondation Ophtalmologique Adolphe de Rothschild, Paris, Île-de-France, France; rblanc@ for.paris

Received 5 March 2020 Revised 22 April 2020

Accepted 29 April 2020 Published Online First 12 June 2020

Check for updates

(C) Author(s) (or their employer(s)) 2020. No commercial re-use. See rights and permissions. Published by BMJ.

To cite: Boisseau W Escalard S, Fahed R, et al. J Neurolntervent Surg 2020;12:1099-1106.

\section{ABSTRACT}

Mechanical thrombectomy is now the standard of care for acute ischemic stroke patients with large vessel occlusions, and can be performed with several devices and techniques. One of these techniques, direct aspiration (DA), consists of navigating a large-bore catheter up to the face of the clot and initiating forceful suction. This comprehensive review has three objectives: (1) to describe the direct aspiration technique; (2) to present the available evidence regarding predictive factors of DA success and performance compared with other techniques; and (3) to discuss the forthcoming improvements in distal aspiration.

\section{INTRODUCTION}

With the results from recent randomized clinical trials (RCT), ${ }^{12}$ the clinical benefit of mechanical thrombectomy (MT) in patients with acute ischemic stroke and anterior circulation large vessel occlusion (AC-LVO) is now well established. This benefit over best medical therapy alone was in large part due to the achievement of excellent recanalization rates and the demonstration of safety of the thrombectomy procedure. Although MT is now standard of care, there is still ongoing debate regarding the optimal first-line thrombectomy strategy. The most commonly used device in these trials was the stent retriever (SR) and, as a result, it is generally felt to be the first-line device for MT. ${ }^{34}$ Alternative techniques such as a direct aspiration first-pass technique (ADAPT) or direct aspiration (DA) are being increasingly used in current clinical practice. These techniques involve the use of large-bore aspiration catheters that are guided to the proximal end of the thrombus and then put under negative pressure (by using vacuum aspiration systems or a syringe) to produce suction on the thrombus. The clot is thus trapped at the tip or aspirated through the aspiration catheter. ${ }^{5}$

Over the past several years, clinical experiences reported in several non-controlled observational studies ${ }^{67}$ have suggested that compared with SRs, DA could result in faster reperfusion, higher rates of successful reperfusion (defined by a Thrombolysis in Cerebral Infarction (TICI) score of $2 \mathrm{~b}$ or more), lower rates of major complications (including fewer distal emboli ${ }^{8}$ and vessel wall damages ${ }^{9}$ ) and superior cost effectiveness. ${ }^{10}$ Similar results have been reported for basilar artery occlusion $(\mathrm{BAO})^{1112}$ and M2 occlusions. ${ }^{13}{ }^{14}$ However promising these results may seem, they were not confirmed by two recent randomized controlled trials, ASTER ${ }^{15}$ (Contact Aspiration vs Stent Retriever for Successful Revascularization) and COMPASS ${ }^{16}$ (Aspiration Thrombectomy vs Stent Retriever Thrombectomy as First-Line Approach for Large Vessel Occlusion), that only demonstrated similar efficacy and safety.

Notably, DA has also been used in combination with SR. Several techniques using the combination of SR and DA have been described: Solumbra (which refers to Solitaire (Medtronic Neurovascular, Irvine, CA) and Penumbra combination), ${ }^{17}$ ARTS (Aspiration (catheter)-(stent) Retriever Technique for Stroke), ${ }^{18}$ and SAVE (Stent retriever Assisted Vacuum-locked Extraction) are some examples. ${ }^{19}$ Again, although several observational studies have reported promising results with such a combined approach, ${ }^{20}$ no clear superiority over DA has been shown in an RCT. ${ }^{21}$

This comprehensive review has three objectives: (1) to perform a description of the direct aspiration technique; (2) to present the available evidence regarding predictive factors of DA success and performance compared with other techniques; and (3) to discuss the forthcoming improvements in distal aspiration.

\section{TECHNIQUE AND DEVICE EVOLUTIONS OF DIRECT ASPIRATION}

The index experience with aspiration in patients with AC-LVO was published in $2006 .^{22}$ In this prospective single-arm trial, complete or partial recanalization was obtained in only seven of 14 patients and only six patients experienced good functional outcome at 3 months. This first experience was followed by the publication of two other studies in $2008^{23}$ and $2009^{24}$ in which an aspiration catheter was used in combination with a device separator to fragment and remove the clot. However, despite excellent recanalization rates with these devices, clinical results were modest. In the Penumbra Pivotal Stroke Trial, while 82\% of these patients achieved a Thrombolysis In Myocardial Infarction (TIMI) score of 2 or 3, only $25 \%$ of patients had a modified Rankin Scale (mRS) score 
Ischemic Stroke

Table 1 List and characteristics of new generation of large-bore aspiration catheters

\begin{tabular}{|c|c|c|c|c|c|c|}
\hline Product name & Length $(\mathrm{cm})$ & $\begin{array}{l}\text { Proximal OD } \\
\text { (inches) }\end{array}$ & $\begin{array}{l}\text { Proximal ID } \\
\text { (inches) }\end{array}$ & $\begin{array}{l}\text { Distal ID } \\
\text { (inches) }\end{array}$ & FDA approved & CE approvec \\
\hline \multicolumn{7}{|l|}{ PENUMBRA (Alameda, CA) } \\
\hline ACE 68 & 132 & 0.080 & 0.068 & 0.068 & $\mathrm{x}$ & $x$ \\
\hline JET 7 & 132 & 0.085 & 0.072 & 0.072 & $\mathrm{x}$ & Pending \\
\hline \multicolumn{7}{|l|}{ STRYKER (Fremont, CA) } \\
\hline CATALYST 7 & 132 & NA & 0.068 & 0.068 & $x$ & $\mathrm{x}$ \\
\hline VECTA & 132 & 0.085 & 0.071 & 0.071 & $\mathrm{x}$ & $x$ \\
\hline \multicolumn{7}{|c|}{ MICROVENTION (Tustin, CA) } \\
\hline SOFIA 6F FLOW PLUS & $125 / 131$ & 0.0825 & 0.070 & 0.070 & $\mathrm{X}$ & $x$ \\
\hline \multicolumn{7}{|l|}{ MEDTRONIC (Irvine, CA) } \\
\hline REACT 68 & 132 & 0.083 & 0.068 & 0.068 & $\mathrm{x}$ & $x$ \\
\hline REACT 71 & 132 & 0.0855 & 0.071 & 0.071 & $x$ & $x$ \\
\hline
\end{tabular}

$\mathrm{CE}$, European conformity; FDA, US Food and Drug Adminstration; ID, inner diameter; OD, outer diameter.

$\leq 2$ at 90 days. ${ }^{24}$ Missing data (including initial Alberta Stroke Program Early CT Score (ASPECTS), time from onset to recanalization, and procedural time) may have explained the lack of clinical response in some patients despite recanalization.

In the years that followed, technological improvements led to the development of new aspiration catheters with larger distal internal diameter, a softer tip, and better navigability and trackability into the distal anatomy. Together, these features allowed operators to reach the intracranial clot and aspirate it without the need for a separator device, a technique called Forced Aspiration Suction Thrombectomy (FAST). ${ }^{25}$ In 2012,

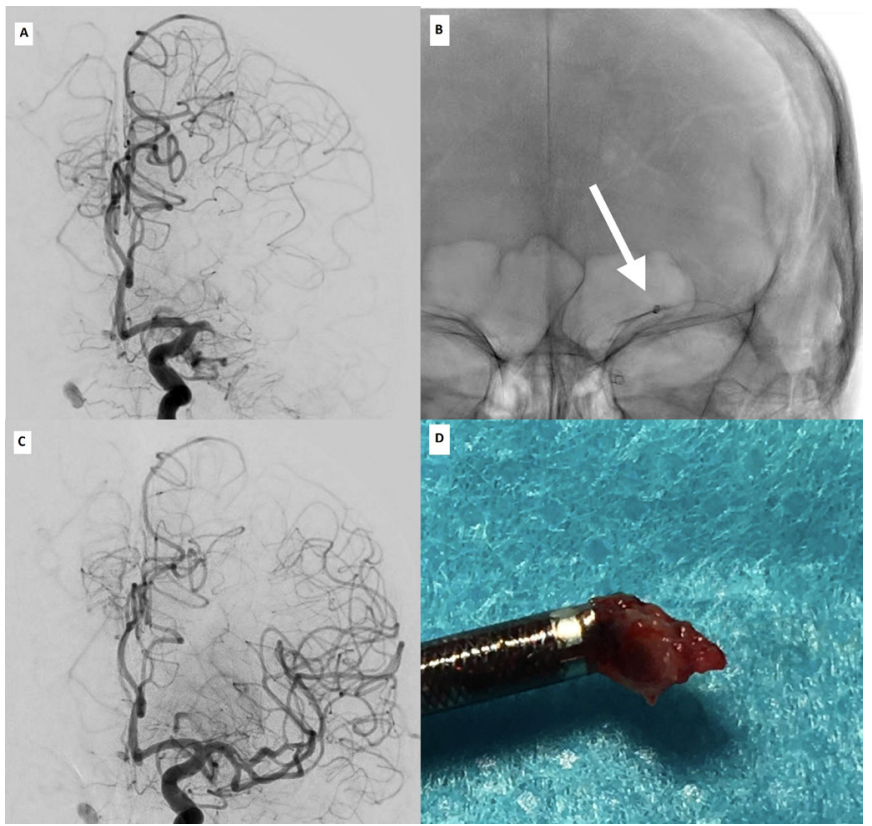

Figure 1 Illustrative case of the direct aspiration technique. Patient presenting 3 hours after acute onset of right-side hemiparesis and aphasia (NIHSS score of 25). Baseline angiography (A) shows total occlusion of the segment M1 of the left MCA. (B) Distal part of the Sofia plus aspiration catheter (white arrow) in contact with the proximal end of the thrombus. (C) Final angiography shows complete reperfusion ( $\mathrm{TICl}$ 3) after first pass. (D) Whole clot is trapped at the tip of the aspiration catheter. MCA, middle cerebral artery; NIHSS, National Institutes of Health Stroke Scale; TICl, Thrombolysis in Cerebral Infarction.
The Randomized Concurrent Controlled Trial to Assess the Penumbra System's Safety and Effectiveness in the Treatment of Acute Stroke (THERAPY trial) began. ${ }^{26}$ THERAPY compared DA plus intravenous tissue plasminogen activator (IV tPA) versus IV tPA alone. This trial was halted early, after the publication of five RCTs confirming the efficacy of MT with SR. Based on the analysis of the 108 patients recruited into this study, patients treated with DA and IV tPA did not achieve better clinical outcomes (mRS 0-2) in comparison with IV tPA alone (38\% vs $30 \%, \mathrm{p}=0.44) .{ }^{26}$ Thus, although there is compelling evidence that MT with SR is superior to best medical therapy, there are no comparable data for DA.

In 2014, a new aspiration technique, ADAPT, emerged. ${ }^{5}$ This technique, using a new generation of large-bore aspiration catheters, has gradually evolved further over subsequent years, with the development of even larger aspiration catheters (a new generation of large-bore aspiration catheters and characteristics are summarized in table 1). In the next paragraph, we delineate a step-by-step description of the DA technique based on our experience and techniques reported in the literature.

A guiding catheter (long introducer sheath or a balloon guide catheter (BGC) for proximal flow arrest) is navigated into the target proximal cervical vessel (carotid or vertebral artery). With the help of a microcatheter and a microwire, a large bore aspiration catheter is then navigated intracranially up to the proximal portion of the clot. Once in place, the aspiration catheter is then connected to an aspiration system such as a pump or a negativepressure syringe. After waiting for a few minutes, the aspiration catheter is then slowly withdrawn (figure 1). Several important features need to be considered here:

- Some of the latest generation aspiration catheters are so flexible that, in some favorable anatomical configurations, they can be navigated without a microcatheter (with a microwire only), or even with no device at all, ${ }^{27}$ which can speed up the procedure. ${ }^{27}$

- When using a microcatheter and/or a microwire to navigate the aspiration catheter, it is recommended to avoid crossing the thrombus whenever possible, as it may be associated with an increased risk of causing distal emboli. Once the aspiration catheter is in contact with the proximal end of the thrombus (within $2-3 \mathrm{~mm}$ of the thrombus if possible), the microcatheter should be removed before initiating suction. ${ }^{28}$ Of note, even an optimal positioning of the aspiration 
catheter, with clear engagement of the clot, does not ensure technical success, as the clot may stay in place.

- There is no consensus regarding the optimal duration after suction activation from which the catheter should be removed. Catheter removal is typically done after $2-3 \mathrm{~min}$ of continuous aspiration.

- Once the aspiration catheter is in place, and the suction activated, it is essential to observe the behavior of the aspiration tube or syringe:

The presence of an immediate backflow could either indicate that the aspiration catheter is not properly placed (usually too proximal to the clot) or the clot has been successfully aspirated (in this case the clot could be seen in the syringe or going through the aspiration tube).

If there is no backflow until the catheter is removed from the patient, it is likely that the clot (or at least a portion of it) is occlusive, inside the catheter or at its tip.

If backflow is restored before the catheter is removed from the long introducer sheath or the BGC, it is likely that the clot was aspirated in the process, or that it was lost on withdrawing the aspiration catheter and remains in the vasculature. In this situation, it is not necessary to completely remove the aspiration catheter, and it may be advantageous to keep the catheter in the patient in the event another pass is required. However, the operator must take great care with the control run as the force of the injection could push residual clot back into the distal vessels.

\section{FACTORS INFLUENCING THE SUCCESS OF DIRECT ASPIRATION}

\section{Technical factors}

A greater aspiration force is one of the most frequently reported factors that may positively influence the success of DA. Several technical factors have been shown to impact aspiration force and, as a result, recanalization. A larger inner diameter (ID) of the aspiration catheter ${ }^{629}$ is a critical factor. For example, the use of ACE 68, which could generate $25 \%$ greater aspiration force than the ACE $60^{629}$ compared with smaller aspiration catheters (ACE 60 and 5Max, Penumbra, Alameda, DA) has been shown to lead to a higher rate of successful reperfusion after the first pass. This was associated with shorter procedural times and lower rates of rescue treatment with SR without increased risk of complications. ${ }^{629}$ Removing the microcatheter before suction has also been shown to influence aspiration force. ${ }^{28}$ Finally, using a vacuum aspiration system with higher aspiration force ${ }^{30}$ has also been beneficial.

In addition to the aspiration force, the use of cyclical aspiration instead of continuous aspiration could result in higher first-pass recanalization rates and lower distal clot embolization. ${ }^{31} 32$ One hypothesis is that cyclical aspiration allows larger clot ingestion into the aspiration catheter and causes less clot fragmentation than continuous aspiration. ${ }^{31}$ Although DA could be used with either a long introducer sheath or a BGC, recent retrospective studies have shown that utilization of a BGC might improve the final and first-pass recanalization rates. ${ }^{33}$ One possible explanation is that inflation of the BGC might reduce the systolic blood pressure acting on the proximal clot face, thus enhancing the effect of aspiration. ${ }^{33}$

\section{Anatomical factors}

The reviewed literature describes the site of arterial occlusion (with isolated middle cerebral artery (MCA) occlusion being the best target for DA), ${ }^{34}$ and the angle of interaction between the aspiration catheter and the clot, ${ }^{35}$ as being important factors for DA success. In one study, an angle of interaction between the aspiration catheter and the clot of $\geq 125.5^{\circ}$ was significantly associated with successful clot removal. ${ }^{35}$ One explanation for this may be that a low angle of interaction between the aspiration catheter and the clot might be associated with a much higher coefficient of friction acting on the thrombus, leading to increased resistance to DA.

\section{Patient factors}

Younger age ${ }^{36}$ and a shorter time from stroke onset to clot contact $^{34}$ have been reported to positively influence the success of aspiration. One speculative explanation for the latter finding could be that a longer delay since stroke onset might promote biological interactions between the thrombus and the arterial wall, leading to a more firm adhesion which could then explain DA failure.

\section{Clot related factors}

Several studies have reported that clot composition (ie, fibrin and red blood cell (RBC) content) could have an impact on the success of aspiration. ${ }^{3738}$ These studies suggest that the DA technique may be more efficient for fibrin-rich clots which could correspond to clots with low density on CT scan and without susceptibility vessel sign (SVS) on MRI. ${ }^{37} 39$ Higher recanalization rates may be achieved with SR for an RBC-rich clot which might correspond to clots with high density on CT scan and with SVS on MRI. ${ }^{3839}$ This hypothesis is to be tested in the VECTOR trial, ${ }^{40}$ an RCT which will compare the combined approach to DA alone as first line for patients with a positive SVS on MRI.

\section{DIRECT ASPIRATION VERSUS OTHER TECHNIQUES \\ Methods}

\section{Literature search}

We performed a literature search through MEDLINE/PubMed and Google Scholar for papers published in the English literature from 2015 to 2020 using the following search key:

"ADAPT", "aspiration", “contact aspiration", "stent", "stent retriever", "acute ischemic stroke", "mechanical thrombectomy", "endovascular treatment", "outcome" and "revascularization". Additional studies were identified through a manual search of the references of published papers and reviews.

Two authors (WB and RB) carried out the literature search and extracted data from relevant studies. Studies comparing clinical and/or angiographic outcomes between DA and SR or the combined approach were included. Exclusion criteria were: (1) case reports; (2) studies not separating outcomes by first-line device use; (3) non-comparative studies (ie, studies with a single group); and (4) studies published before 2015 and the use of new generation of stent retriever and large-bore aspiration catheters.

\section{Outcome variables}

For the purposes of this study, patients were divided into three groups according to the first-line device use: SR, DA, and the combination of both techniques. The following outcomes were studied: good functional outcome defined as an mRS $\leq 2$ at 90 days following endovascular treatment, mortality, successful recanalization defined as TICI $2 \mathrm{~b} / 3$, complete recanalization (TICI 3), first pass recanalization rates defined as complete recanalization with a single thrombectomy device pass, and procedure duration (ie, delay between puncture and recanalization). Results were divided into three groups according to the occlusion location: AC-LVO (intracranial internal carotid artery, M1 or 


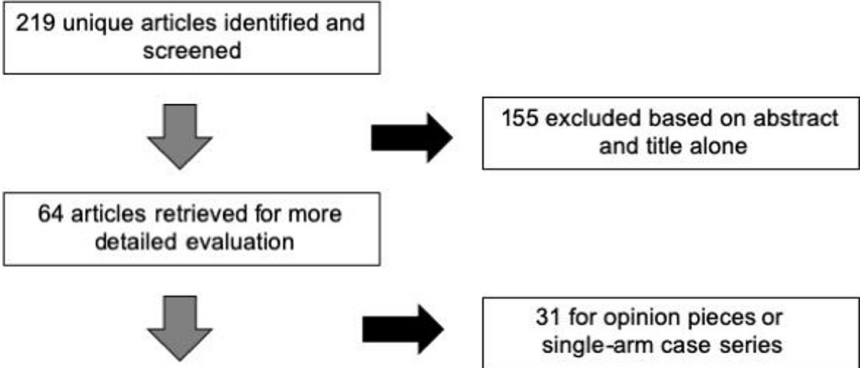

33 studies included in the study

Figure 2 PRISMA flow diagram. PRISMA, Preferred Reporting Items for Systematic Reviews and Meta-Analyses.

proximal M2 branches of the MCA), basilar artery, and medium vessel occlusions (MeVOs) (ie, distal M2 or M3 segments of the MCA, pericallosal artery or the posterior cerebral artery). ${ }^{41}$

\section{RESULTS}

A total of 219 articles were retrieved after the first round of research, 155 of which were excluded by title or abstract screening. The full texts of the remaining 64 articles were accessed, and 33 articles matching the inclusion criteria were included: 26 studies with AC-LVO (including 20 studies comparing DA and SR and six comparing DA and the combined approach), six studies with $\mathrm{BAO}$, and one study with MeVOs. The search selection process is illustrated in figure 2 .

\section{Anterior circulation large vessel occlusion}

Direct aspiration versus stent retriever first-line technique

The 15 selected studies included five observational studies, ${ }^{7}$ 42-45 two RCTs (ASTER ${ }^{15}$ and COMPASS ${ }^{16}$ ) and eight systematic reviews and meta-analyses. ${ }^{46-53}$ Study characteristics are summarized in online supplementary table 1 . Devices, and clinical and angiographic outcomes, are depicted in table 2 .

The majority of these studies demonstrated similar successful (TICI $2 \mathrm{~b}$ or more) and complete (TICI 3) reperfusion rates, both after the first-line strategy and at the end of the procedure. ${ }^{151642-44}$ Successful reperfusion rates ranged from 42-83\% after first-line DA, and from $78-92 \%$ at the end of the procedure. ${ }^{7} 15164244$ These studies also reported similar adverse events rates (including symptomatic intracranial hemorrhage $(\mathrm{sICH})$ and embolization in a new territory (ENT)) and clinical outcomes with both techniques. ${ }^{7} 151642$ Nevertheless, several studies $^{74} 45$ and meta-analyses ${ }^{49} 52$ have suggested that the proportion of patients who needed rescue therapies was higher among patients who received first-line DA. A similar result was found in the ASTER trial in which $32.8 \%$ of patients treated with DA received rescue treatment compared with $23.8 \%$ in the SR group (OR 1.57, 95\% CI 0.99 to $2.47 ; \mathrm{p}=0.05) .{ }^{15}$

Finally, studies have suggested several advantages of DA over SR as a first-line strategy. Total device cost seems to be significantly lower with DA. In the recent COMPASS trial, the use of DA as first-line approach led to a mean reduction of $\$ 5074$ in the cost of devices used. ${ }^{16}$ DA was also associated with faster groin-to-reperfusion time. ${ }^{1642-45}$ According to the ASTER and COMPASS trials, DA resulted in a shorter mean procedural time of 7-10 min compared with SR. ${ }^{1516}$ Despite this time advantage, DA was not associated with improved patient outcomes. Similar results have been reported in studies focusing on patients with proximal M2 occlusions only (three observational studies and two meta-analyses) (see table 3$).{ }^{54-58}$

It should be noted that several of the aforementioned studies included patients before 2015, at a time when MT was not yet standard of care; thus these studies reflect data accrued with older generations of devices, with older techniques and without the rigorous patient selection criteria responsible for the great success in recent trials. Furthermore, these early studies were plagued with a significant heterogeneity in thrombectomy technique which confounds comparison between SR and DA. In some studies, the concomitant use of a DA in the stent retriever firstline group was frequent. In the COMPASS trial ${ }^{16} 85 \%$ of patients in the SR group also received DA, as did $55.7 \%$ of SR patients in the study by Stapleton et l $^{44}$ In the ASTER trial, only $2.9 \%$ of the SR group also had DA. ${ }^{15}$ As a consequence, many SR patients were not in fact SR-only patients but rather patients treated with a combined approach. In similar fashion, the use of BGCs with SR was not standardized in several studies, introducing another level of heterogeneity. ${ }^{7} 1516$ 42-44 While BGCs were systematically used with SR in the ASTER trial, ${ }^{15}$ they were used between $0-72 \%$ of cases in other studies. ${ }^{7642-44}$ Finally, many DA studies included patients treated with intermediate-size aspiration catheters (which, as described above, might not be as efficient as larger-bore aspiration catheters). Only the COMPASS ${ }^{16}$ study used predominantly large-bore aspiration catheters (with ID $\geq 0.064$ inches). In COMPASS, ${ }^{16} 98 \%$ of patients in the DA firstline group were treated with large-bore catheters compared with only $47.7 \%$ in the ASTER study. ${ }^{15}$ Although future RCTs will be required to confirm these findings, it is reasonable to speculate that better reperfusion rates could have been reached with the use of these larger-bore aspiration catheters.

\section{Direct aspiration versus combined approach}

Ten studies compared DA alone versus the combined treatment (combination of DA and SR) in patients with AC-LVO. Six of these studies used the combined approach as a rescue treatment (after the failure of first-line DA) and not as first-line technique and were thus excluded.

Our review thus includes four observational studies ${ }^{10} 205960$ and one $\mathrm{RCT}^{21}$ a non-inferior randomized multicenter study comparing the effects of a three-dimensional stent retriever in conjunction with local aspiration-based mechanical thrombectomy versus aspiration-based thrombectomy alone as a primary modality for endovascular stroke intervention. Three of these studies ${ }^{102021}$ have been pooled in a recent meta-analyses. ${ }^{61}$ Study characteristics are summarized in online supplementary table 1. Details of the revascularization devices as well as the clinical and angiographic outcomes are reported in table 4.

According to this recent meta-analysis, ${ }^{61}$ combined treatment was associated with better successful reperfusion rates (OR 1.47, 95\% CI 1.02 to 2.12 ) compared with DA alone; however, there was no difference in clinical outcomes (favorable outcome OR 0.84, $95 \%$ CI 0.38 to 1.86 ). One study ${ }^{59}$ further investigated this result. After stratification according to the occlusion site, the combined approach was more efficient for carotid " $T$ " occlusions with higher rates of recanalization (TICI $2 \mathrm{c}$ or more $55.2 \%$ vs $15.4 \%$; $\mathrm{p}=0.025)$, with higher rates of first-pass mTICI $2 \mathrm{c}$ or more $(59.6 \%$ vs $33.3 \% ; \mathrm{p}=0.019)$, and demonstrated a trend toward better final successful reperfusion rate $(93.1 \%$ vs $65.4 \% ; \mathrm{p}=0.10) .{ }^{59}$ For M1 segment occlusion, similar reperfusion rates were reported $(94.8 \%$ vs $83.3 \% ; p=0.187)$. Finally, two observational studies have also reported a lower rate of ENT with combined treatment but also an increased risk of sICH, ${ }^{20} 60$ a difference that was not found in the RCT. ${ }^{21}$ There is an ongoing RCT which may help to confirm 


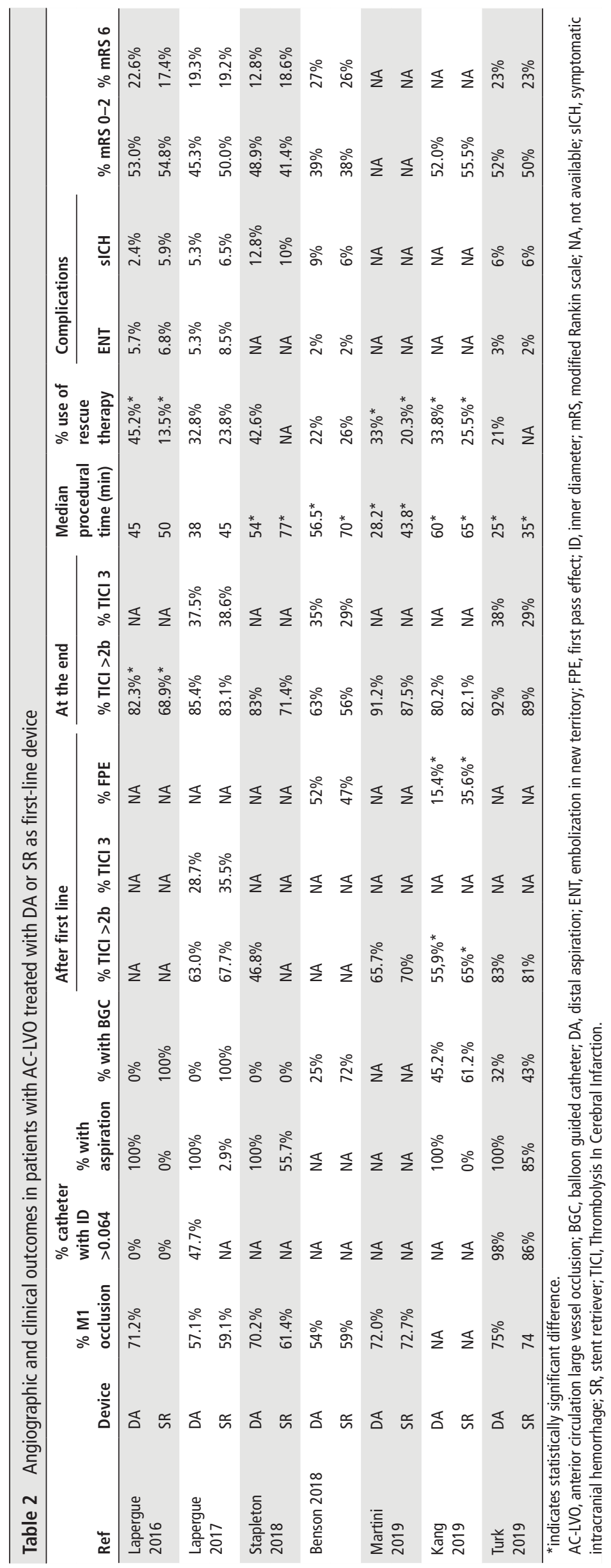




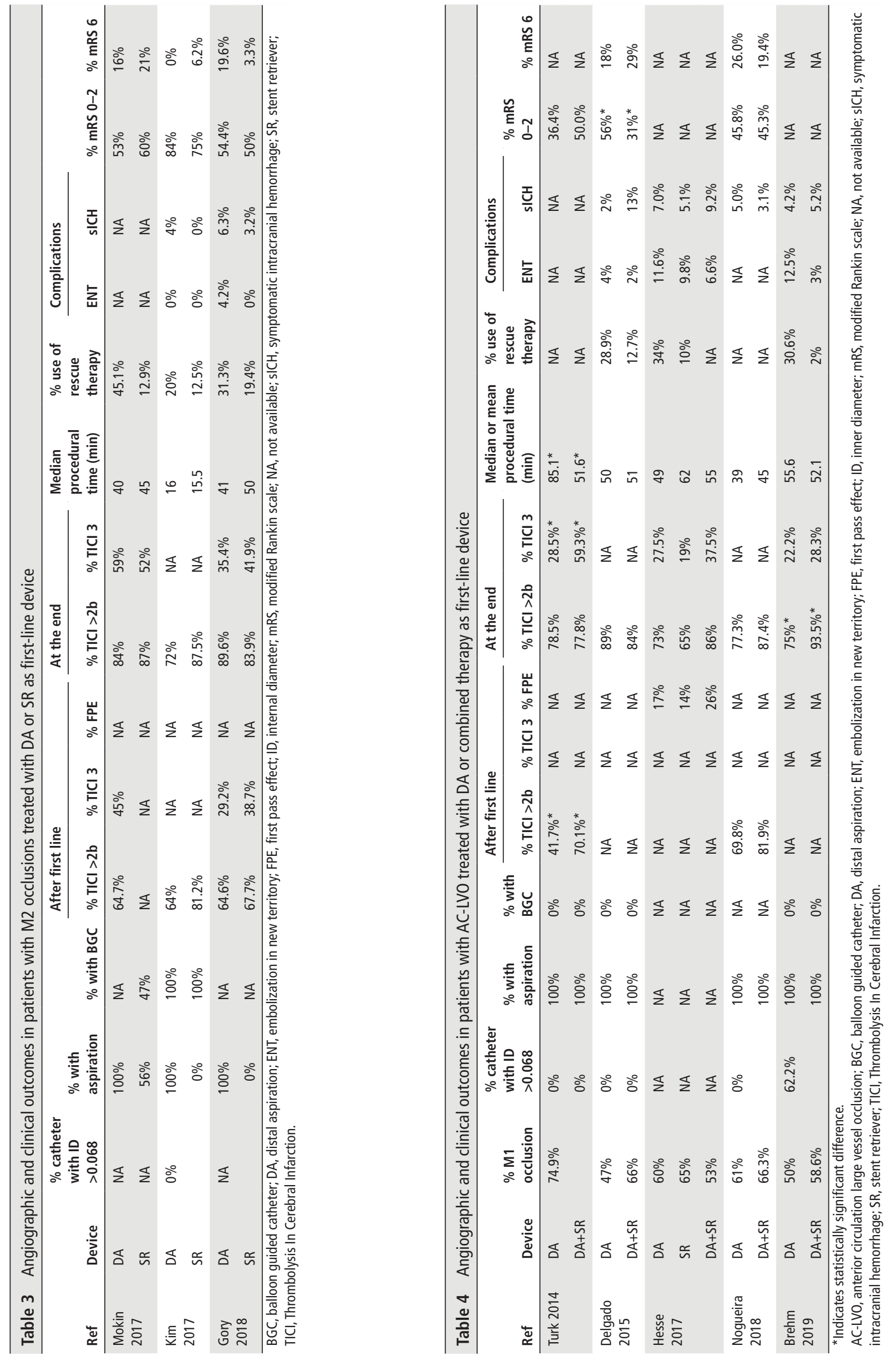


the potential superiority for the combined approach in carotid " $T$ " occlusions as well as provide more information on the potential increased hemorrhagic risk of combined treatment. ${ }^{62}$

\section{Medium vessel occlusions}

Only one study has directly compared DA and SR as the first-line approach in patients with MeVOs. ${ }^{14}$ In this retrospective study of 137 patients, the use of a $3 \mathrm{~mm}$ Trevo stent (Stryker Neurovascular, Fremont, CA) compared to Penumbra 3MAX device (Penumbra, Alameda, CA) resulted in higher rates of first-pass mTICI $2 b-3$ reperfusion and lower utilization of adjuvant therapy. ${ }^{14}$

\section{Basilar artery occlusions}

Five studies, including two prospective registries with retrospective analysis $^{12} 63$ and two retrospective studies, ${ }^{11} 6465$ have compared DA and SR approaches as first-line strategies in patients with BAOs. These five studies are summarized in a recent meta-analysis. ${ }^{66}$ The pooled results suggested that the DA approach achieved a significantly higher rate of successful recanalization (OR 2.0, 95\% CI 1.1 to $3.5 ; \mathrm{p}=0.02$ ) with a lower ENT rate compared with first-line SR. ${ }^{66} \mathrm{~A}$ similar trend was found for the mTICI 3 rate. ${ }^{66}$ Again, procedure duration was significantly lower with DA than SR. ${ }^{66}$ However, once again, these angiographic outcomes were not associated with better clinical outcomes. ${ }^{66}$ No significant difference for hemorrhagic complications, functional independence or mortality at 3 months was found between the two techniques.

\section{CHALLENGES AND FUTURE PROSPECTS}

New, larger aspiration catheters and vacuum aspiration systems with higher aspiration force are under development. The Penumbra JET 7 catheter (Penumbra, Alameda, CA) (with 0.072 inch ID), AXS Vecta (Stryker Neurovascular, Fremont, CA), the React catheter (Medtronic Neurovascular, Irvine, CAA) (with 0.071 inch ID) and the Penumbra Jet Engine (Penumbra, Alameda, CA) have been recently approved by the US Food and Drug Administration. ${ }^{30} 67$ It is, however, important to note that the largest aspiration catheters (JET 7, Vecta and React 071) are not compatible with current BGCs. A novel aspiration catheter system, the R4 Q aspiration catheter (MIVI Neuroscience, Inc, Eden Prairie, MN) has a larger proximal ID, and in a recent benchtop study demonstrated a substantial increase in aspirated flow rate and suction force compared with the commercially available standard tubular catheters. ${ }^{68}$ As a final example, the Advanced Thrombectomy System (ANCD) is a novel stroke thrombectomy device incorporating a coated self-expanding funnel. Theoretically, this funnel must be deployed after SR expansion to the proximal part of the clot and might restrict the flow and enhance the aspiration force. Altogether, this new device might facilitate clot retrieval and reduce clot fragmentation. ${ }^{69}$ The combination of these new generation aspiration catheters and vacuum aspiration systems may produce a higher aspiration force and tip suction forces and further improve recanalization rates with DA. ${ }^{30}$ Although this new generation of large-bore aspiration catheters appears to be efficient and safe based on recent non-controlled observation studies, ${ }^{70-72}$ concerns exist regarding the navigability and trackability of these devices, especially around the ophthalmic artery in tortuous vessels. For example, the use of Vecta ${ }^{72}$ or React ${ }^{71}$ catheters required the use of an SR to deliver the aspiration catheter up to the clot level (the "grappling hook technique") in about $25 \%$ of cases. By comparison, this "grappling hook technique" was required in 40\% of cases with the ACE 068. Further studies are needed to better investigate the efficiency and safety of this new generation of aspiration catheters and vacuum aspiration systems.
Although the benefit of MT in MeVOs is suggested in several non-controlled observational studies (mainly in patients with M2 occlusions), these findings need to be confirmed in RCTs. ${ }^{41}$ MeVOs (M2 or M3 segments of the MCA, pericallosal artery or the posterior cerebral artery) can lead to severe AIS. While these smaller vessels can still cause a severe deficit, thrombectomies in these vascular beds raise some questions. The risk of vessel perforation is perceived to be higher given the smaller caliber of artery, yet it is actually unknown. It is also unclear whether medical therapy is superior to endovascular therapy in terms of clinical outcomes. Once again, the use of DA will need to be assessed in this specific scenario.

Finally, given the several limitations in the aforementioned cited literature and the rapid evolution of suction equipment, further randomized trials are required in order to better assess the efficiency of DA compared with SR or the combined approach. The research community may want to reconsider the optimal outcome metric for evaluating thrombectomy devices. The three trials mentioned above have used three different outcomes as their primary endpoint. A clinical outcome was used in COMPASS (clinical functional outcome at 90 days) and an angiographic outcome was used in both ASTER (mTICI $2 \mathrm{~b}$ or 3 ) and the trials by Nogueira et al (mTICI 2 or 3). Clinical outcome after MT depends on multiple factors besides recanalization, including, but not limited to, patient age, comorbidities, initial infarct volume and occurrence of sICH. Moreover, although improvements in thrombectomy devices have led to a sharp increase in successful reperfusion rates, clinical outcomes have remained largely unchanged. For example, the recent COMPASS trial reported successful reperfusion in $90 \%$ of enrolled patients, yet the percentage of patients with functional independence at 90 days is only $50 \% .^{16}$ Thus clinical outcomes do not appear to be the optimal endpoint to capture the efficacy of thrombectomy devices. Although angiographic outcomes may represent a useful surrogate endpoint, the definition of successful revascularization should be reviewed. Recent RCTs have reported successful reperfusion (mTICI $\geq 2 b$ ) in up to $90 \%$ of enrolled patients. ${ }^{16}$ Given these current rates of revascularization, if we use the somewhat broad "mTICI $2 \mathrm{~b}$ or more" as the primary outcome to judge new devices, it may be unrealistic to expect higher rates of successful recanalization. Perhaps stricter parameters such as near complete (mTICI 2c or 3), complete reperfusion (mTICI 3$)^{73}$ or first pass effect ${ }^{7475}$ rates should be used as the new standard definition for technical success in future trials.

\section{CONCLUSION}

Direct aspiration is a fast, safe and efficient thrombectomy technique that deserves its place in the interventional neuroradiology armamentarium. Given the rapid evolution of DA equipment and the inherent limitations to the many studies gathered in our review, we believe further randomized trials are needed to better capture the true efficiency of DA compared with SR and the combined approach. Rates of near complete recanalization and first pass effect should be used as the new standard definitions for technical success in these future trials.

\footnotetext{
Author affiliations

${ }^{1}$ Interventional Neuroradiology, Fondation Ophtalmologique Adolphe de Rothschild, Paris, île-de-France, France

${ }^{2}$ Department of Medicine - Division of Neurology, Ottawa Hospital, Ottawa, Ontario, Canada

${ }^{3}$ Neurology Department, Hôpital Foch, Suresnes, Île-de-France, France

${ }^{4}$ Université de Paris, Paris, Île-de-France, France
} 
${ }^{5}$ Department of Medicine - Division of Neurology, Royal Columbian Hospital, New Westminster, British Columbia, Canada

${ }^{6}$ Diagnostic and Interventional Neuroradiology, Centre Hospitalier Universitaire de Nancy, Nancy, France

${ }^{7}$ Neurology Stroke Unit, University Hospital Centre Nancy, Nancy, France

Correction notice Since the online publication of this article, the Provenance and peer review statement was updated from 'Not commissioned; externally peer reviewed' to 'Commissioned; externally peer reviewed.'

Contributors Study design: WB, SE, RF, RB. Acquisition, analysis, or interpretation of data: WB, SE, RF, RB. Drafting of the manuscript: All authors. Supervision: RB.

Funding The authors have not declared a specific grant for this research from any funding agency in the public, commercial or not-for-profit sectors.

Competing interests None declared.

Patient consent for publication Not required.

Provenance and peer review Commissioned; externally peer reviewed.

\section{ORCID iDs}

Robert Fahed http://orcid.org/0000-0002-1887-5097

Stanislas Smaida http://orcid.org/0000-0002-6486-1710

François Delvoye http://orcid.org/0000-0002-0697-2156

Benjamin Gory http://orcid.org/0000-0001-8424-4464

Michel Piotin http://orcid.org/0000-0002-1354-4328

Raphaël Blanc http://orcid.org/0000-0002-3975-3865

\section{REFERENCES}

1 Berkhemer OA, Fransen PSS, Beumer D, et al. A randomized trial of intraarterial treatment for acute ischemic stroke. N Engl J Med 2015;372:11-20.

2 Bracard S, Ducroca X, Mas JL, et al. Mechanical thrombectomy after intravenous alteplase versus alteplase alone after stroke (THRACE): a randomised controlled trial. Lancet Neurol 2016:15:1138-47.

3 Powers WJ, Rabinstein AA, Ackerson T, et al. Guidelines for the early management of patients with acute ischemic stroke: 2019 update to the 2018 guidelines for the early management of acute ischemic stroke: a guideline for healthcare professionals from the American Heart Association/American Stroke Association. Stroke 2019:50:STR.0000000000000211

4 Turc G, Bhogal P, Fischer U, et al. European Stroke Organisation (ESO) - European Society for Minimally Invasive Neurological Therapy (ESMINT) guidelines on mechanical thrombectomy in acute ischemic stroke. J Neurointerv Surg 2019:neurintsurg-2018-014569

5 Turk AS, Spiotta A, Frei D, et al. Initial clinical experience with the ADAPT technique: a direct aspiration first pass technique for stroke thrombectomy. J Neurointerv Surg 2014:6:231-7

6 Delgado Almandoz JE, Kayan Y, Wallace AN, et al. Larger ACE 68 aspiration catheter increases first-pass efficacy of ADAPT technique. J Neurointerv Surg 2019;11:141-6.

7 Lapergue B, Blanc R, Guedin P, et al. A direct aspiration, first pass technique (ADAPT) versus stent retrievers for acute stroke therapy: an observational comparative study. AJNR Am J Neuroradiol 2016;37:1860-5.

8 Mokin M, Setlur Nagesh SV, lonita CN, et al. Comparison of modern stroke thrombectomy approaches using an in vitro cerebrovascular occlusion model. AJNR Am J Neuroradiol 2015;36:547-51.

9 Peschillo S, Diana F, Berge J, et al. A comparison of acute vascular damage caused by ADAPT versus a stent retriever device after thrombectomy in acute ischemic stroke: a histological and ultrastructural study in an animal model. J Neurointerv Surg 2017:9:743-9

10 Turk AS, Campbell JM, Spiotta A, et al. An investigation of the cost and benefit of mechanical thrombectomy for endovascular treatment of acute ischemic stroke. $J$ Neurointerv Surg 2014;6:77-80.

11 Son S, Choi DS, Oh MK, et al. Comparison of solitaire thrombectomy and penumbra suction thrombectomy in patients with acute ischemic stroke caused by basilar artery occlusion. J Neurointerv Surg 2016;8:13-18.

12 Gory B, Mazighi M, Blanc R, et al. Mechanical thrombectomy in basilar artery occlusion: influence of reperfusion on clinical outcome and impact of the first-line strategy (ADAPT vs stent retriever) 2018:129:1482.

13 Premat K, Bartolini B, Baronnet-Chauvet F, et al. Single-center experience using the $3 \mathrm{MAX}$ reperfusion catheter for the treatment of acute ischemic stroke with distal arterial occlusions. Clin Neuroradiol 2018:28:553-62.

14 Haussen DC, Eby B, Al-Bayati AR, et al. A comparative analysis of 3MAX aspiration versus $3 \mathrm{MM}$ Trevo retriever for distal occlusion thrombectomy in acute stroke. J Neurointerv Surg 2020;12:neurintsurg-2019-014990

15 Lapergue B, Blanc R, Gory B, et al. Effect of endovascular contact aspiration vs stent retriever on revascularization in patients with acute ischemic stroke and large vessel occlusion: the ASTER randomized clinical trial. JAMA 2017;318:443-52.

16 Turk AS, Siddiqui A, Fifi JT, et al. Aspiration thrombectomy versus stent retriever thrombectomy as first-line approach for large vessel occlusion (COMPASS): a multicentre, randomised, open label, blinded outcome, non-inferiority trial. Lancet 2019:393:998-1008.

17 Humphries W, Hoit D, Doss VT, et al. Distal aspiration with retrievable stent assisted thrombectomy for the treatment of acute ischemic stroke. J Neurointerv Surg 2015;7:90-4.

18 Massari F, Henninger N, Lozano JD, et al. ARTS (Aspiration-Retriever Technique for Stroke): initial clinical experience. Interv Neuroradiol 2016;22:325-32.

19 Maus V, Behme D, Kabbasch C, et al. Maximizing first-pass complete reperfusion with SAVE. Clin Neuroradiol 2018;28:327-38.

20 Hesse AC, Behme D, Kemmling A, et al. Comparing different thrombectomy techniques in five large-volume centers: a 'real world' observational study. J Neurointerv Surg 2018;10:525-9.

21 Nogueira RG, Frei D, Kirmani JF, et al. Safety and efficacy of a 3-dimensional stent retriever with aspiration-based thrombectomy vs aspiration-based thrombectomy alone in acute ischemic stroke intervention: a randomized clinical trial. JAMA Neurol 2018;75:304-11.

22 Imai K, Mori T, Izumoto $\mathrm{H}$, et al. Clot removal therapy by aspiration and extraction for acute embolic carotid occlusion. AJNR Am J Neuroradiol 2006;27:1521-7.

23 Bose $\mathrm{A}$, Henkes $\mathrm{H}$, Alfke $\mathrm{K}$, et al. The penumbra system: a mechanical device for the treatment of acute stroke due to thromboembolism. AJNR Am I Neuroradiol 2008;29:1409-13.

24 Penumbra Pivotal Stroke Trial Investigators. The Penumbra Pivotal Stroke Trial: safety and effectiveness of a new generation of mechanical devices for clot removal in intracranial large vessel occlusive disease. Stroke 2009;40:2761-8.

25 Kang D-H, Hwang Y-H, Kim Y-S, et al. Direct thrombus retrieval using the reperfusion catheter of the Penumbra system: forced-suction thrombectomy in acute ischemic stroke. AJNR Am J Neuroradiol 2011;32:283-7.

26 Mocco J, Zaidat 00 , von Kummer $R$, et al. Aspiration thrombectomy after intravenous alteplase versus intravenous alteplase alone. Stroke 2016;47:2331-8.

27 Heit JJ, Wong JH, Mofaff AM, et al. Sofia intermediate catheter and the SNAKE technique: safety and efficacy of the Sofia catheter without guidewire or microcatheter construct. J Neurointerv Surg 2018;10:401-6.

28 Nikoubashman O, Alt JP, Nikoubashman A, et al. Optimizing endovascular stroke treatment: removing the microcatheter before clot retrieval with stent-retrievers increases aspiration flow. J Neurointerv Surg 2017:9:459-62.

29 Alawieh A, Chatterjee AR, Vargas J, et al. Lessons learned over more than 500 stroke thrombectomies using ADAPT with increasing aspiration catheter size. Neurosurgery 2020;86:61-70.

30 Yaeger K, Iserson A, Singh P, et al. A technical comparison of thrombectomy vacuum aspiration systems. J Neurointerv Surg 2020;12:72-6.

31 Arslanian RA, Marosfoi M, Caroff J, et al. Complete clot ingestion with cyclical ADAPT increases first-pass recanalization and reduces distal embolization. J Neurointerv Surg 2019;11:931-6.

32 Simon S, Grey CP, Massenzo T, et al. Exploring the efficacy of cyclic vs static aspiration in a cerebral thrombectomy model: an initial proof of concept study. J Neurointerv Surg 2014;6:677-83

33 Kang D-H, Kim BM, Heo JH, et al. Effect of balloon guide catheter utilization on contact aspiration thrombectomy. J Neurosurg 2018;131:1494.

34 Blanc R, Redjem H, Ciccio G, et al. Predictors of the aspiration component success of a direct aspiration first pass technique (ADAPT) for the endovascular treatment of stroke reperfusion strategy in anterior circulation acute stroke. Stroke 2017;48:1588-93.

35 Bernava G, Rosi A, Boto J, et al. Direct thromboaspiration efficacy for mechanical thrombectomy is related to the angle of interaction between the aspiration catheter and the clot. J Neurointerv Surg 2019:neurintsurg-2019-015113.

36 Mascitelli JR, Kellner CP, Oravec CS, et al. Factors associated with successful revascularization using the aspiration component of ADAPT in the treatment of acute ischemic stroke. J Neurointerv Surg 2017;9:636-40.

37 Ye G, Cao R, Lu J, et al. Association between thrombus density and reperfusion outcomes using different thrombectomy strategies: a single-center study and metaanalysis. Front Neurol 2019:10:843.

38 Bourcier R, Mazighi M, Labreuche J, et al. Susceptibility vessel sign in the ASTER trial: higher recanalization rate and more favourable clinical outcome after first line stent retriever compared to contact aspiration. J Stroke 2018:20:268-76.

39 Liebeskind DS, Sanossian N, Yong WH, et al. CT and MRI early vessel signs reflect clot composition in acute stroke. Stroke 2011:42:1237-43.

40 Janot K, Zhu F, Kerleroux B, et al. "Adaptative endovascular strategy to the CloT MR in large intracranial vessel occlusion" (VECTOR): study protocol of a randomized control trial. J Neuroradiol 2019.

41 Goyal M, Ospel JM, Menon BK, et al. MeVO: the next frontier? I Neurointerv Surg 2020:neurintsurg-2020-015807.

42 Bernsen MLE, Goldhoorn R-JB, van Oostenbrugge RJ, et al. Equal performance of aspiration and stent retriever thrombectomy in daily stroke treatment. J Neurointerv Surg 2019;11:631-6.

43 Martini M, Mocco J, Turk A, et al. 'Real-world' comparison of first-line direct aspiration and stent retriever mechanical thrombectomy for the treatment of acute ischemic stroke in the anterior circulation: a multicenter international retrospective study. $J$ Neurointerv Surg 2019;11:957-63 
44 Stapleton CJ, Leslie-Mazwi TM, Torok CM, et al. A direct aspiration first-pass technique vs stentriever thrombectomy in emergent large vessel intracranial occlusions. J Neurosurg 2018;128:567-74.

45 Kang D-H, Kim JW, Kim BM, et al. Need for rescue treatment and its implication: stent retriever versus contact aspiration thrombectomy. J Neurointerv Surg 2019;11:979-83.

46 Gory B, Armoiry X, Sivan-Hoffmann R, et al. A direct aspiration first pass technique for acute stroke therapy: a systematic review and meta-analysis. Eur J Neurol 2018;25:284-92.

47 Phan K, Dmytriw AA, Teng I, et al. A direct aspiration first pass technique vs standard endovascular therapy for acute stroke: a systematic review and meta-analysis. Neurosurgery 2018;83:19-28.

48 Qin C, Shang K, Xu S-B, et al. Efficacy and safety of direct aspiration versus stent-retriever for recanalization in acute cerebral infarction: a PRISMA-compliant systematic review and meta-analysis. Medicine 2018;97:e12770-e70.

49 Tsang COA, Cheung IHW, Lau KK, et al. Outcomes of stent retriever versus aspirationfirst thrombectomy in ischemic stroke: a systematic review and meta-analysis. AJNR Am J Neuroradiol 2018;39:2070-6.

50 Wei D, Mascitelli JR, Nistal DA, et al. The use and utility of aspiration thrombectomy in acute ischemic stroke: a systematic review and meta-analysis. AJNR Am J Neuroradiol 2017;38:1978-83.

51 Boulanger M, Lapergue B, Turjman F, et al. First-line contact aspiration vs stentretriever thrombectomy in acute ischemic stroke patients with large-artery occlusion in the anterior circulation: systematic review and meta-analysis. Interv Neuroradiol 2019;25:244-53.

52 Hsieh KL-C, Chuang K-I, Weng H-H, et al. First-line a direct aspiration first-pass technique vs. first-line stent retriever for acute ischemic stroke therapy: a metaanalysis. Front Neurol 2018;9:801-01.

53 Primiani CT, Vicente AC, Brannick MT, et al. Direct aspiration versus stent retrieve thrombectomy for acute stroke: a systematic review and meta-analysis in 9127 patients. J Stroke Cerebrovasc Dis 2019;28:1329-37.

54 Kim Y-W, Son S, Kang D-H, et al. Endovascular thrombectomy for M2 occlusions: comparison between forced arterial suction thrombectomy and stent retriever thrombectomy. J Neurointerv Surg 2017;9:626-30.

55 Mokin M, Primiani CT, Ren Z, et al. Endovascular treatment of middle cerebral artery M2 occlusion strokes: clinical and procedural predictors of outcomes. Neurosurgery 2017;81:795-802

56 Gory B, Lapergue B, Blanc R, et al. Contact aspiration versus stent retriever in patients with acute ischemic stroke with $\mathrm{M} 2$ occlusion in the ASTER randomized trial (contact aspiration versus stent retriever for successful revascularization). Stroke 2018;49:461-4

57 Phan K, Maingard J, Kok HK, et al. Contact aspiration versus stent-retriever thrombectomy for distal middle cerebral artery occlusions in acute ischemic stroke: meta-analysis. Neurointervention 2018;13:100-9.

58 Saber H, Narayanan S, Palla M, et al. Mechanical thrombectomy for acute ischemic stroke with occlusion of the M2 segment of the middle cerebral artery: a metaanalysis. J Neurointerv Surg 2018;10:620-4.
59 Brehm A, Maus V, Tsogkas I, et al. Stent-retriever assisted vacuum-locked extraction (SAVE) versus a direct aspiration first pass technique (ADAPT) for acute stroke: data from the real-world. BMC Neurol 2019;19:65.

60 Delgado Almandoz JE, Kayan Y, Young ML, et al. Comparison of clinical outcomes in patients with acute ischemic strokes treated with mechanical thrombectomy using either Solumbra or ADAPT techniques. J Neurointerv Surg 2016:8:1123-8.

61 Texakalidis P, Giannopoulos S, Karasavvidis T, et al. Mechanical thrombectomy in acute ischemic stroke: a meta-analysis of stent retrievers vs direct aspiration vs a combined approach. Neurosurgery 2019;372:nyz258.

62 Lapergue B, Labreuche J, Blanc $\mathrm{R}$, et al. Combined use of contact aspiration and the stent retriever technique versus stent retriever alone for recanalization in acute cerebral infarction: the randomized aster 2 study protocol. J Neurointerv Surg 2020:12:471-6.

63 Kang D-H, Jung C, Yoon W, et al. Endovascular thrombectomy for acute basilar artery occlusion: a multicenter retrospective observational study. J Am Heart Assoc 2018;7:e009419.

64 Gerber JC, Daubner D, Kaiser D, et al. Efficacy and safety of direct aspiration first pass technique versus stent-retriever thrombectomy in acute basilar artery occlusion-a retrospective single center experience. Neuroradiology 2017;59:297-304.

65 Mokin M, Sonig A, Sivakanthan S, et al. Clinical and procedural predictors of outcomes from the endovascular treatment of posterior circulation strokes. Stroke 2016:47:782-8

66 Ye G, Lu J, Qi P, et al. Firstline a direct aspiration first pass technique versus firstline stent retriever for acute basilar artery occlusion: a systematic review and metaanalysis. J Neurointerv Surg 2019;11:740-6.

67 Gross BA, Jadhav AP, Jovin TG, et al. Clinical comparison of new generation 0.071inch and 0.072-inch aspiration catheters. World Neurosurg 2019;130:e463-6.

68 Long TD, Kallmes DF, Hanel R, et al. Novel aspiration catheter design for acute stroke thrombectomy. J Neurointerv Surg 2019;11:190-5.

69 Sanchez S, Cortiñas I, Villanova H, et al. Ancd thrombectomy device: in vitro evaluation. J Neurointerv Surg 2020;12:77-81.

70 Hassan A, Fifi J, Zaidat O. Efficacy and safety of mechanical thrombectomy using larger bore jet 7 aspiration catheters for intracranial large vessel occlusion. J Neurolntervent Surg 2019;11:A126-A26.

71 Raymond SB, Nasir-Moin M, Koch MJ, et al. Initial experience with react 68 aspiration catheter. Interv Neuroradiol 2020:159101991989892 (published Online First: 2020/01/24).

72 Satti S, Sivapatham T, Almallouhi E, et al. Vecta 071 and 074 large bore aspiration catheter: initial multi-center experience. J Neurolntervent Surg 2019:11:A114-5.

73 Rizvi A, Seyedsaadat SM, Murad MH, et al. Redefining 'success': a systematic review and meta-analysis comparing outcomes between incomplete and complete revascularization. J Neurointerv Surg 2019;11:9-13.

74 Zaidat 00, Castonguay AC, Linfante I, et al. First pass effect: a new measure for stroke thrombectomy devices. Stroke 2018;49:660-6.

75 Ducroux C, Piotin M, Gory B, et al. First pass effect with contact aspiration and stent retrievers in the aspiration versus stent retriever (ASTER) trial. J Neurointerv Surg 2020;12:neurintsurg-2019-015215 